\title{
त्री \\ Proliferación subdisciplinar en biología, debacle del reduccionismo y nuevas estrategias de unificación
} María José FERREIRA Guillermo Folguera

\begin{abstract}
茴
RESUMEN

Durante las últimas décadas, la biología ha sido objeto de una considerable proliferación teórica y subdisciplinar, hecho que suscita la pregunta acerca de su unidad. Por ello, a partir del abandono del reduccionismo como estrategia unificadora, el interrogante filosófico es qué otras formas alternativas de relaciones subdisciplinares son posibles, y si dichas relaciones logran dar unidad a la biología. En el presente artículo, a partir de una breve consideración de algunos de los problemas que el programa reduccionista ha tenido en biología, analizaremos diferentes tipos de relaciones que se encuentran entre las subdisciplinas de la biología contemporánea. En particular, indagaremos cuatro de las principales propuestas que pueden encontrarse en la literatura filosófica especializada; ofrecemos entonces dos nuevas propuestas de relación interdisciplinar: el isomorfismo de la biología evolutiva, e la importación/exportación de cuerpos teóricos, propia de la relación entre la ecología y la fisiología. Finalmente, presentaremos algunas consideraciones en torno al objetivo de la unidad de la ciencia en general, como también acerca de la clase de cohesión que creemos posible para la biología a partir de nuestros análisis, defendiendo la idea de un pluralismo de las relaciones interdisciplinares y de la unidad como un fenómeno local.
\end{abstract}

Palabras-clave • Unidad de la ciencia. Reduccionismo. Relaciones interdisciplinares.

Relaciones subdisciplinares. Filosofía de la biología. Ecología.

\section{INTRODUGGIÓN: GRISIS DEL REDUGGIONISMO \\ COMO ESTRATEGIA DE UNIFIGAGIÓN Y BIOLOGÍA}

El ideal de unificación de la ciencia ha sido entendido y buscado de muy diversas maneras. Basta recordar que el proyecto moderno de la mathesis universalis buscó unificar las ciencias bajo el método de la razón misma. Ya en el siglo xx, el Círculo de Viena tuvo como uno de sus objetivos principales lograr la unificación de las ciencias mediante una estrategia que incluyó la construcción de un lenguaje formal neutral. Más recientemente, a mediados del siglo pasado, la estrategia elegida para lograr la unidad fue el 
intento de reducir determinados cuerpos teóricos a otros, uno de cuyos representantes más importantes fue Ernst Nagel. Desde entonces, sin embargo, esta estrategia no estuvo exenta de numerosas dificultades. Estos inconvenientes no sólo se han hecho presentes en la relación entre las ciencias naturales (física, química y biología), sino también incluso al seno de cada una de estas disciplinas.

A su vez, otro proceso que acompañó el siglo xx fue el de la aparición y consolidación de nuevas áreas del conocimiento. En particular, una de las ciencias en las cuales se reconoce una mayor proliferación subdisciplinar en las últimas décadas fue la biología. Piénsese en el origen y consolidación de áreas tales como genética molecular, biología molecular, ecología del paisaje, macroecología, neurobiología, evo-devo, genómica, por sólo nombrar algunas de esas nuevas disciplinas. Dicha proliferación conllevó a que la pregunta relativa a la unificación sea sumamente significativa también hacia el interior de la biología. Sin embargo, luego de las dificultades que ha presentado la estrategia reduccionista, las preguntas filosóficas apuntan a las características de las nuevas estrategias de unificación entre subdisciplinas al interior de la biología.

En el presente trabajo nos proponemos analizar críticamente algunos de los principales tipos de relaciones subdisciplinares que han sido reconocidos desde la filosofía de la biología, así como proponer otras relaciones entre campos del saber que a nuestro entender son fundamentales en la biología contemporánea. Con este fin, el trabajo posee la siguiente estructura. En la sección 1, presentaremos y analizaremos algunas de las principales dificultades que ha tenido la reducción como estrategia de unificación dentro de la biología. En la sección 2, indagaremos algunas de las principales estrategias de relaciones subdisciplinares ofrecidas por la filosofía de la biología. Posteriormente, en la sección 3, propondremos otras dos relaciones subdisciplinares alternativas a las reductivas de gran relevancia en la biología de la actualidad. Por último, la sección 4 está destinada a señalar algunos apuntes finales referidos a la cuestión de si dichas estrategias alternativas lograrían cumplir con el proyecto de unidad de las áreas de conocimiento, así como dirigidos a comprender mejor los argumentos a favor y en contra de la búsqueda de una unificación entre las diferentes subdisciplinas que forman parte de la biología.

\section{REDUGGIÓN Y UNIDAD EN LA BIOLOGÍA}

Tal como fue adelantado en la sección anterior, durante el siglo xx la reducción fue la estrategia privilegiada para lograr la unidad de la ciencia desde la filosofía de la ciencia. Sin embargo, el reduccionismo como estrategia de unificación presentó numerosos problemas. Dado que "reducción” se dice de muchas maneras, previo a presentar 
algunas de sus principales dificultades, conviene aclarar qué entenderemos aquí por reducción. En principio, nuestro análisis se focalizará en la denominada "reducción epistemológica” o "teórica” (cf. Ayala, 1983; Dobzhansky et al., 1980; Mayr, 1988), cuya versión clásica fue desarrollada principalmente por Nagel, que define la reducción como "la explicación de una teoría o un conjunto de leyes experimentales establecidas en un área de investigación, a través de una teoría general, aunque no siempre de un dominio diferente" (Nagel, 1961, p. 338). En palabras de Ayala, la reducción que se da cuando "las teorías y leyes experimentales formuladas en un campo de la ciencia pueden considerarse casos especiales de teorías y leyes formuladas en algún otro campo científico", en cuyo caso "la primera rama de la ciencia ha sido reducida a la segunda" (Ayala, 1983, p. 12).

En términos más precisos, de acuerdo con este modelo, una teoría $T_{1}$ se reduce a otra teoría $T_{2}$ si y sólo si las leyes de $T_{1}$ pueden ser deducidas de las leyes de $T_{2}$ con la ayuda de algunos postulados adicionales (también llamados "principios puente"). Para realizar una reducción, deben satisfacerse dos condiciones formales: conectabilidad y derivabilidad. La primera exige que para cada término en $T_{1}$ exista un término correspondiente en $T_{2}$. La segunda, y dada la conectabilidad, las leyes de $T_{1}$ deben poder ser derivadas a partir de las leyes de $T_{2}$, junto con ciertos principios puente.

El modelo clásico de reducción presentó problemas de diversa índole. Primeramente, no ha logrado aplicarse en casos científicamente relevantes. Además, supone concebir las teorías como conjuntos axiomáticos interpretados, cuando no sólo es infrecuente que las teorías logren ser axiomatizadas de una manera interesante, sino que, además, se ha criticado que las relaciones entre teorías sean relaciones lógicas (Primas, 1998). Tampoco es del todo claro cuál es la naturaleza de los principios puente, nucleares al modelo nageliano.

Además de la filosofía general de la ciencia, las filosofías especiales también han ofrecido diversos argumentos contra este modelo de reducción interteórica. En particular, la tradición reduccionista como posible estrategia para la unidad de la biología, tampoco está libre de críticas y problemas. Por un lado, en biología no pueden reconocerse grandes éxitos del programa en sus diferentes versiones; por ejemplo, el modelo Nagel-Schaffner, siendo uno de los que ha recibido mayores esfuerzos, no se pudo aplicar exitosamente para reducir la genética clásica a la genética molecular. A su vez, numerosos investigadores han marcado problemas insalvables para la reducción como estrategia de unificación. Por ejemplo, Ingo Brigandt (2011) sostiene que las críticas al reduccionismo proceden típicamente del hecho de que las clases de un campo de nivel alto y de un campo de nivel bajo mantienen relaciones muchos-muchos, y que, por ello, no se puede obtener ninguna correspondencia sistemática entre los conceptos de ambas teorías. La justificación de ello radica en que lo que en un campo de nivel más 
alto es una clase o un fenómeno unificado, puede consistir para un campo de nivel más bajo en diferentes clases o fenómenos, a la vez que una clase o fenómeno de nivel bajo puede corresponder a diferentes clases o fenómenos más altos. A su vez, Pigliucci y Kaplan (2006) consideran que aunque se pueda explicar de un modo reduccionista cómo algo hace lo que hace, esto no equivale a explicar lo que algo es; y esta es una distinción que el reduccionismo no puede recoger. Todas estas dificultades han llevado a numerosos pensadores a dudar en el éxito de dicha empresa, tanto en lo relativo a las relaciones disciplinares como entre subdisciplinas relativas al estudio de lo viviente (cf. Wimsatt, 1976; Darden \& Maull, 1977; Kitcher, 1984; Rosenberg, 1985, 1994; Dupré, 1993; Burian, 1996; Caponi, 2004; Brigandt \& Love, 2008). Finalmente, esta crisis del reduccionismo ha sido considerada por diversos autores como un aspecto positivo, a partir de que establece un tipo de relación fuertemente asimétrica entre áreas del conocimiento, lo cual podría tener aparejado consecuencias no deseables como, por ejemplo, ciertos efectos limitantes en lo que respecta a su proliferación teórica.

Sin embargo, en el marco general del empirismo lógico de mediados de siglo xx, tal como hemos adelantado, el reduccionismo presentaba sin dudas un aspecto de gran atractivo, a saber, una estrategia clara y general de búsqueda de la unidad. Las características de dicha estrategia lo hacen especialmente interesante frente a escenarios de gran complejidad, tal como el caso de la gran proliferación subdisciplinar que se ha dado al seno de la biología en las últimas décadas. Ahora bien, frente a la crisis del reduccionismo como estrategia de unificación, ¿pueden reconocerse nuevas estrategias de relaciones subdisciplinares al seno de la biología? Y en tal caso, ¿qué características presentan las mismas? En la siguiente sección abordaremos estos interrogantes tratando de reconocer y problematizar otros tipos de relaciones interteóricas entre subdisciplinas que se encuentren actualmente dentro de la biología según la bibliografía especializada.

\section{ANÁLISis Grítico de GUATro estrategias}

\section{DE RELAGIONES SUBDISGIPLINARES ALTERNATIVAS A LA REDUGGIÓN}

El objetivo de buscar cierta unificación al seno de la biología, en general, suele ser justificado a los fines de evitar que las diferentes áreas que indagan la vida presenten desconexiones entre sí. Por ello, los problemas de la reducción han llevado a buscar otras estrategias de integraciones entre subdisciplinas. A continuación analizaremos cuatro de las propuestas más prominentes que pueden reconocerse en la biología contemporánea y que han sido analizadas desde la filosofía de la biología. La primera es la denominada "teorías intercampos", propuesta por Lindley Darden y Nancy Maull 
en 1977. La segunda pone el eje de integración en los mecanismos y fue elaborada por Darden en los últimos años. En tercer lugar, indagaremos la relación de refinamiento conceptual propuesta por Philip Kitcher en 1984. Finalmente, será analizada la propuesta, aún más reciente, de Alan Love relativa a las “agenda de problemas”.

\subsection{TEORÍAS INTERCAMPoS}

Darden y Maull (1977) propusieron una alternativa a la reducción en términos de teorías que vinculan dos campos entre sí. Contra las visiones centradas en teorías, esta propuesta consideró como objeto de relaciones a los campos (fields), noción más amplia que la de teoría, ya que estos pueden contener más de una teoría o, incluso, ninguna. Desde esta perspectiva, una teoría intercampo (inter-field theory) surge cuando es necesario hacer explícita una relación entre dos campos, o bien cuando estos comparten un interés común en diferentes aspectos de un mismo fenómeno del que ninguno por separado (con sus conceptos y métodos) puede dar cuenta. Así, el aporte de una teoría intercampo es una nueva idea pertinente para los campos que vincula. En principio, una teoría de esta clase no reduce los campos que vincula, sino que cada uno de ellos mantiene su independencia, y se ve favorecido por el puente trazado. Entre las funciones que desempeña una teoría intercampo pueden señalarse las siguientes: logra responder preguntas surgidas en un campo que no pueden responderse sólo con los elementos de ese campo, permite rescatar ítems previamente relegados del dominio de uno o de ambos campos, consigue extender la capacidad predictiva de los campos hacia nuevos ítems y, finamente, genera nuevas líneas de investigación. Uno de los ejemplos en los que las autoras se basan es el de cómo la teoría cromosómica de la herencia mendeliana vinculó a la citología y a la genética. En principio estas dos áreas compartían el interés por la herencia, pero la primera no tenía métodos para investigar la función de los cromosomas (la localización propuesta para el material hereditario) en la producción de características individuales heredables, mientras que la segunda no podía responder a la pregunta por la localización de los genes. La teoría cromosómica de la herencia mendeliana trazó un puente entre genética y citología al postular que los genes están en los cromosomas, lo cual constituye una idea nueva. A su vez, esta teoría logró explicar las semejanzas entre genes y cromosomas, resolvió los problemas que en cada campo por sí sólo no podía resolver, permitió además predecir nuevos ítems para el dominio de ambos campos, y generó nuevas líneas de investigación. Sin embargo, cabe señalar que Darden y Maull no han tenido pretensiones de aplicar este análisis a todos los casos, es decir, a cualesquiera campos y teorías. 


\subsection{INTEGRAGión MEDIANTE MEGANISMOS}

Si bien el modelo señalado en la sección anterior relaciona campos, lo hace mediante una teoría, con lo cual, la propuesta tiene sus limitaciones en la medida que no en todo campo hay una y sólo una teoría, tal como lo señalaron Darden y Maull. Más recientemente, Darden (2005) ha propuesto una relación entre sub disciplinas biológicas mediante el análisis filosófico de sus mecanismos. Peter Machamer, Lindley Darden y Carl Craver (2000) han definido "mecanismos" como entidades y actividades que están relacionadas de manera tal que producen un cambio regular desde las condiciones iniciales a las condiciones terminales. Para llevar a cabo este análisis, hay que explicar la relación que se da entre los mecanismos de cada uno, esto es, entre sus respectivos inputs y outputs, ítems (partes) componentes, y sus contextos. Por ejemplo, Darden analiza la relación entre la genética clásica y la genética molecular: mientras que la primera se centra en la meiosis como el período especial en el ciclo de las células germinales y las entidades relevantes en tal mecanismo son los cromosomas, la segunda da cuenta de la expresión génica, lo que significa que se trata de otro mecanismo, en tanto concierne a un período diferente en el ciclo celular y tiene lugar en todos los tipos de células, y sus entidades relevantes están por debajo del nivel de los cromosomas (segmentos de ADN, codones etc.). Claramente, la explicación ofrecida desde la genética clásica no se reduce a la explicación propia de genética molecular, en la medida en que ambas dan cuenta de diferentes mecanismos en diferentes períodos temporales. El análisis filosófico, entonces, explica la relación que se da entre ambas subdisciplinas, detallando precisamente que la expresión génica y la meiosis son diferentes periodos de un proceso celular total, y que las diferentes entidades involucradas en cada caso mantienen relaciones de parte-todo o de interacción entre sí.

\subsection{Refinamiento gongeptual}

Philip Kitcher (1984) ha propuesto, entre otras, la relación de refinamiento conceptual. Una teoría posterior provee un refinamiento conceptual de la teoría anterior cuando la posterior da una especificación de las entidades que pertenecen a la extensión de predicados en el lenguaje de la teoría anterior. De esto resulta que se altera el modo como se fijan los referentes de esos predicados de acuerdo con las nuevas especificaciones teóricas. Este refinamiento puede ocurrir de dos maneras; o bien cuando una nueva teoría suministra una caracterización de la extensión de un predicado para el cual no había ninguna caracterización previa, o bien cuando la nueva teoría ofrece una nueva caracterización que hace necesario corregir la anterior. Kitcher ejemplifica esta relación con el fenómeno de la mutación en genética clásica y en genética molecular; 
en particular, considera que una perspectiva molecular permite responder de modo general qué es una mutación. El referente del predicado "alelo mutante" se fijó, inicialmente, mediante la descripción "segmento cromosómico que produce una desviación fenotípica heredable" (Kitcher, 1984, p. 364). Con el descubrimiento del crossingover desigual, fue evidente para los genetistas clásicos que la primera caracterización cubría tanto los casos en que la estructura interna del gen era alterada, así como casos en los que los genes vecinos eran transpuestos. Por ello, fue necesario usar una descripción menos aplicable: “segmento cromosómico que produce una desviación fenotípica heredable como resultado del cambio interno dentro de un alelo" (Kitcher, 1984, p. 364). De este modo, puesto que la genética molecular ofrece explicaciones precisas de estos cambios internos, se ofrece en esta subdisciplina una caracterización de la mutación todavía más informativa, a saber, los alelos mutantes son segmentos de ADN que resultan de los alelos anteriores mediante la eliminación, inserción o sustitución de nucleótidos. Así, esta re-fijación de los referentes permite distinguir casos de mutación de casos de recombinación, y así resolver controversias que se suscitaron en la genética clásica. Además, la genética molecular provee refinamientos significativos de los conceptos de "gen" y "alelo".

\subsection{Agenda de PRoblemas}

Alan Love (2005, 2008, 2010) desliza la cuestión de las disciplinas a los problemas. Para ello, se vale de la noción de "agenda de problemas" (problem agenda), que es una unidad erotética, es decir, una unidad compuesta por preguntas que guardan relaciones entre sí y que están asociadas a ciertos estándares de adecuación explicativa. Son estas unidades las que determinan qué campos son relevantes para resolver el problema y qué contribuciones intelectuales se necesitan de cada campo. Dada su estructura interna, un problema agenda anticipa también cómo las contribuciones de los diferentes campos deben coordinarse e integrarse en la explicación total. Son los problemas los que pueden estructurar la integración. Así, algunos de los problemas científicos motivan una investigación interdisciplinaria, lo cual es apropiadamente representado mediante la noción de problema agenda, que es compatible con la pluralidad de metas explicativas en biología.

Sin embargo, es claro que según Love una unificación completa de la biología está fuera de nuestro alcance. Love ejemplifica este modelo con el origen de la innovación (novelty) evolutiva, que constituye un problema central para la biología evolutiva, y del que la biología evolutiva neo-darwiniana tradicional no ha podido dar cuenta, pues estudia el cambio cuantitativo en las frecuencias génicas de las poblaciones mediante el favorecimiento de ciertas variantes ya existentes, pero no cómo se originan 
estas variantes en primer lugar. En la actualidad, es claro que el problema de la innovación requiere contribuciones de muchos campos (filogenia, paleontología, biología del desarrollo, morfología).

Para Love, la explicación del origen de la innovación es una agenda de problemas que determina cómo deben coordinarse las distintas contribuciones de los campos biológicos. Primero, hay que exponer una secuencia de rasgos morfológicos relevantes en diferentes especies de un linaje hasta la presencia de la novedad. Para este componente del problema, la paleontología debe proveer los datos fósiles de las estructuras estudiadas y la filogenia debe aportar el árbol evolutivo de las especies involucradas. Luego, hay que dar cuenta del problema de un modo causal-mecánico, y aquí la biología del desarrollo debe explicar cómo los sistemas de desarrollo ancestrales fueron modificados para resultar en un sistema cambiado, produciendo la estructura cualitativamente novedosa; entre otros aportes necesarios.

En una línea similar, Ingo Brigandt (2010, 2011) llama la atención sobre las metas o agendas explicativas de los distintos campos en la biología. Considera que debe distinguirse una agenda explicativa de una explicación; mientras que una explicación es una representación del fenómeno estudiado por la ciencia, una agenda explicativa es acerca de la ciencia misma, son valores de los científicos. Las agendas explicativas contribuyen a la identidad intelectual de los acercamientos científicos, y las distintas agendas explicativas de los diferentes campos biológicos dan cuenta de la diversidad dentro de la biología. La coexistencia de diferentes metas explicativas puede obstaculizar la comunicación entre las diferentes subdisciplinas, y resultar conflictiva cuando el mismo fenómeno es abordado con diferentes metas explicativas por diferentes científicos. Sin embargo, al mismo tiempo, cabe reconocer que el hecho de que una misma meta sea compartida por diferentes campos puede motivar y coordinar la investigación interdisciplinaria fructíferamente.

A continuación, tal como anticipamos, propondremos dos formas de relación entre áreas de conocimiento biológico que ofrecen cierta integración y que, creemos, no han sido atendidas por la filosofía de la biología.

\section{Propuesta de nuevas ReLaGiones subdisciplinares presentes en LA BIOLOGÍA CONTEMPORÁNEA: EXPORTAGIÓN/IMPORTAGIÓN E ISOMORFISMO}

\subsection{EXPORTAGIÓN/IMPORTAGIÓN DE GUERPOS TEÓRIGOS}

La primera de las relaciones subdisciplinares que propondremos se hace presente en el caso en el que un área adopta un cuerpo teórico (o una "parte" de él) proveniente de 
otra para explicar sus propios fenómenos. Este tipo de relación subdisciplinar encuentra un ejemplo de gran actualidad: el escenario dado por la macroecología, y la fisiología, mediante la teoría metabólica. Para comprender este caso hay que recordar que en el último tercio del siglo xx fueron revisados algunos de los aspectos fundamentales de la ecología disciplinar. En particular, a fines de la década de 1980 se originó la denominada macroecología, centrada en estudios comparativos entre taxones de muy alta jerarquía (Ginzburg \& Colyvan, 2004). Los estudios macroecológicos llevan a cabo una indagación comparativa de las especies relacionadas filogenéticamente, garantizando así su pertenencia al mismo gremio ecológico (Brown \& Maurer, 1987; 1989). Inicialmente, la macroecología se trató meramente de un conjunto de fenómenos; es decir, no presentó ningún tipo de marco teórico definido capaz de dar cuenta de sus propios patrones; por lo cual, los patrones generados a partir del registro de las variables eran explicados mediante procesos tales como la extinción diferencial, la especiación, la colonización o las restricciones energéticas (Brown \& Maurer, 1987). Podríamos decir que puesto que no hubo una teoría propiamente macroecológica, ni tampoco explicaciones propiamente macroecológicas. Gaston y Blackburn (2000) adjudican la ausencia de cuerpo teórico al hecho de que la macroecología era una disciplina todavía muy reciente.

Sin embargo, en la última década del siglo xx fue incorporado un marco conceptual en la subdisciplina. En efecto, a partir de las investigaciones realizadas por Brown y colaboradores, la macroecología importó la denominada "teoría metabólica de la ecología" (TME) como conjunto teórico de la macroecología. La TME fue desarrollada a partir del análisis de las tasas metabólicas, susceptibles de ser estudiadas en condiciones experimentales dentro del laboratorio (cf. Di Pasquo \& Folguera, 2009). En términos generales podemos resumir a la TME a partir de dos hipótesis principales. La primera constituye, en rigor, un componente de dominio fisiológico, pues se trata de la relación entre el tamaño corporal, la temperatura corporal y el metabolismo de los organismos. La segunda, ya a escala macroecológica, relaciona el metabolismo de los organismos con el flujo energético y con el flujo de materia de las poblaciones, las comunidades y los ecosistemas (Brown et al., 2004). De este modo, puede verse que, en términos generales, a partir de una combinación de los efectos de estas variables (tamaño corporal, temperatura corporal y metabolismo de los individuos - nivel fisiológico-) es posible dar cuenta de la estructura y la dinámica de la ecología de sistemas (organizaciones de nivel más alto, como la ecología de poblaciones, de comunidades y de ecosistemas) (Brown et al., 2004).

El punto clave de la macroecología está dado por esta última, pues constituye una vinculación entre las escalas macro y micro (en este caso el nivel fisiológico). Además, la incorporación de este marco teórico permitió explicaciones de causas proximales. 
Si bien es cierto que en algunos análisis que hemos realizado anteriormente (cf. Di Pasquo \& Folguera, 2009) hemos reconocido también cierto intento reductivo acoplado a la importación del cuerpo teórico (debido en buena medida a la ambigüedad de las expresiones empleadas por los autores, que por momentos parecen querer reducir las relaciones macroecológicas a los valores fisiológicos de los organismos involucrados), este no constituye un verdadero caso de reduccionismo interteórico. En efecto, no se trata, en este caso, de una relación entre dos teorías distintas, pues la macroecología no contaba con ninguna teoría previa; no había ninguna teoría que pudiera ser reducida por la teoría importada ni conectada con ella mediante principios puente.

\subsection{IsOMORFISMo}

Otra forma de relación subdisciplinar, con un lugar significativo en la biología contemporánea a nuestro entender, es la que hemos denominado "isomorfismo". No nos estamos refiriendo aquí a su homónimo matemático, sino que esta terminología refiere a la consideración de que (al menos) dos áreas presentan entidades y/o procesos similares, pero que se ubican en diferentes áreas biológicas. Es decir, hay isomorfismo cuando en dos subdisciplinas diferentes son identificadas entidades y procesos que presentan ciertas similitudes sustantivas. Esa forma compartida por dos campos de conocimiento hace posible una integración que no resulta reductiva en un sentido epistemológico, en la medida en que no se deducen las leyes de una teoría a las leyes de otra, ni aun ontológico, porque lejos de reducirse una entidad de mayor nivel a una de nivel inferior, esta relación muestra que se multiplican entidades y procesos similares a los del nivel inferior en el nivel más alto.

Uno de los casos más emblemáticos de este tipo de relación ha sido la propuesta de una jerarquía selectiva por parte de varios autores, propuesta en décadas pasadas por investigadores de la talla de Stephen Gould, Niles Eldredge, Ian Tatersall, entre otros. En términos generales, la propuesta de estos autores presentó dos elementos significativos. Por un lado, la multiplicación de las unidades de selección en la propuesta jerárquica. Por otro lado, en todos los niveles se propuso la acción de mecanismos "alternativos" completamente análogos a la selección natural microevolutiva. Estas jerarquías genealógicas propuestas estuvieron comprometidas con la búsqueda de entidades individuales que pertenecieran a los diferentes niveles de la jerarquía, acudiendo a una analogía con el organismo microevolutivo; es decir, entidades de niveles suprapoblacionales pasaron a ser consideradas como los individuos de esos niveles. Es interesante notar que pese a que se reivindica la autonomía de los niveles macroevolutivos, estos se explican por explícita referencia a los niveles microevolutivos y mediante analogías con de los conceptos utilizados en el ámbito microevolutivo. 
Proliferagión subdisgiplinar en biología, debagle del RedugGionismo...

No analizaremos aquí el éxito de la propuesta de Gould y otros, sólo nos hemos limitado a señalar cómo dicha conformación isomórfica parece haber generado un tipo de relación novedosa respecto al tipo de relación reductiva. En este caso, la estructura isomórfica está dada por una conceptualización que se da tanto de entidades (individuos) como mecanismos (selección) que tienen su origen en un campo determinado (microevolución en el ejemplo analizado) y que es aplicado al resto de los ámbitos (evolutivos en el ejemplo).

\section{4 ¿POR QUÉ LA UNIDAD? \\ ¿QUÉ UNIDAD ES POSIBLE PARA LA BIOLOGÍA?}

En el presente trabajo hemos partido de la actitud generalizada de desacreditar las empresas reduccionistas para, en una segunda instancia, presentar y analizar cuatro alternativas no-reduccionistas de relaciones interdisciplinares que se encuentran en la literatura filosófica en relación con la biología. Posteriormente, hemos presentado y desarrollado otras dos propuestas propias, que a nuestro entender ocupan un lugar significativo en la biología de la actualidad.

A partir del presente recorrido se suscita naturalmente la pregunta ¿por qué buscar la unidad? La filosofía ha ofrecido varias y muy distintas respuestas a este interrogante. En relación con el contexto que aquí nos ocupa, hay que notar que la forma más típica de reacción al fracaso del reduccionismo fue pluralista, ya sea en sentido metodológico, ontológico, teórico o explicativo; entendiendo, en general, que la biología consiste en y necesita de una diversidad de campos, métodos, teorías, explicaciones. En este sentido, y semejantemente a Rosenberg (cf. 1994), Dupré (cf. 1983; 1993) sostuvo la idea de la des-unidad de la ciencia en un mundo inherentemente desordenado y atacó el reduccionismo valiéndose principalmente de ejemplos tomados de la ecología y de la genética mendeliana. La razón principal para la irreductibilidad entre disciplinas (o ciencias) es que ellas requieren diferentes clasificaciones, las cuales constituyen una parte inextricable de las disciplinas. Además, las clases (en el sentido laxo en que Dupré las entiende) son muy heterogéneas en biología. Kitcher (cf. 1999), si bien reconoce que la unificación de la biología es imposible, la postula como un ideal regulativo al que los biólogos deben aspirar tanto como puedan.

Contra estas argumentaciones, Bechtel (cf. 1986; 2006) sostiene que la creación de nuevas disciplinas conduce tanto a la integración como a la especialización o desintegración; de modo que cuánta integración y qué tipo de combinación de integración y especialización se necesita depende y varía con el problema científico considerado. Contra Kitcher, afirma que la unificación no es un objetivo en sí mismo, sino 
algo necesario para la resolución de un determinado problema. En una línea similar, Brigandt (cf. 2010; 2011) propone una idea de unidad más moderada, en tanto admite que se necesita alguna integración en ciertos problemas biológicos, pero esta no es un objetivo universal de los científicos. Incluso otros problemas pueden ser resueltos sin ninguna integración, y más aún, cierta especialización en diferentes disciplinas puede conducir a resolver problemas complejos.

En lo que a nuestras propuestas concierne, cabe señalar que hemos arribado a tales estrategias a partir de la pregunta por la relación entre subdisciplinas en determinados escenarios teóricos que se han dado en las últimas décadas. En este sentido, consideramos que, así como hemos podido analizar y tipificar la relación entre la fisiología y la macroecología, por una parte, y la microevolución y macroevolución, por la otra, existen otras formas en que distintas subdisciplinas biológicas comportan cierta integración. Por ello, nos interesa subrayar el hecho de que las necesidades de los casos concretos tienen que guiar la búsqueda del tipo de integración satisfactoria. Que haya propuestas tan variadas y atractivas en esta materia (tal como las seis aquí analizadas) motiva, a nuestro entender, el abandono de un modelo de relación universal y nos habilita a hablar de un pluralismo de las relaciones interdisciplinares, entendiendo por esto el hecho de que no hay un modelo o una estrategia de integración universalmente aplicable en biología.

Es relevante a nuestros fines, entonces, preguntarnos qué tipo de unidad se busca cuando se relacionan distintos campos de conocimiento de un modo no reduccionista, porque mientras que la reducción se consideraba, en principio, posible para todos los casos (dado que se insistía en considerar a toda teoría como un conjunto axiomático interpretado), lo que reconocemos en las propuestas no reduccionistas aquí presentadas es que estas formas de relaciones tienen como resultado, cuando mucho, una unificación puramente local. Las seis propuestas que aquí hemos considerado consisten en un análisis de casos específicos, y no vemos que exista ninguna pretensión, por parte de sus autores, de extenderlas o generalizarlas; antes bien, estos se muestran escépticos respecto de la posibilidad de dar con un modelo de relación global que vincule a todas las subdisciplinas biológicas entre sí.

Greemos que estas formas de relación logran, en efecto, concretar unidad entre áreas del saber, pero a condición de restringir la noción de unidad a una cualidad local, particular, y práctica, en el sentido de que no se logra teorizando un modelo abstracto de unificación independientemente de los casos, sino lidiando con casos y problemas concretos. Y por esta razón, se trata de una unidad a la que se llega por modos diversos; en efecto, todos los tipos de relaciones subdisciplinares que hemos presentado (tanto aquellos cuatro de la bibliografía específica como los dos que hemos esbozado) son estrategias igualmente válidas de unidad pese a sus importantes diferencias de forma. 


\section{Conclusiones}

Quizás podamos concluir con la idea de que la unificación continúa siendo un objetivo, pero sólo para casos concretos y entendiéndola en este sentido modesto y restringido. No obstante, no hay, al menos por ahora, razones suficientes para pensar que una unificación completa en biología (en sentido distinto al del reduccionista) sea en principio imposible, y en ese sentido, consideramos que la cuestión continúa en buena medida abierta. Sin embargo, puesto que tampoco es evidente que una empresa semejante pueda llevarse a cabo, creemos que la actitud más interesante por el momento consiste en dar un paso hacia el pluralismo. Más aún, la adopción de un punto de vista pluralista conlleva a un mayor desarrollo tanto de las subdisciplinas como de las propuestas y concreciones de zonas de integración local entre ellas, mientras que la búsqueda de una unificación global podría constreñir este desarrollo. Integración local y pluralismo de las relaciones subdisciplinares son las notas que, a nuestro juicio, parecen describir mejor la situación actual de las subdisciplinas en biología, como también prescribir una dirección fructífera para ulteriores investigaciones. En cualquier caso, serán los problemas concretos los que indiquen de qué manera se deben articular nuestros conocimientos acerca de la vida.@

María José Ferreira Grupo de Filosofía de la Biología,

Facultad de Filosofía y Letras, Facultad de Ciencias Exactas y Naturales, Universidad de Buenos Aires, Argentina. mariaferreiraruiz@gmail.com

\section{Guillermo Folguera}

Facultad de Ciencias Exactas y Naturales,

Universidad de Buenos Aires, Consejo Nacional de Investigaciones Científicas y Técnicas, Argentina. guillefolguera@yahoo.com.ar

Proliferation of subdisciplines in biology: the debacle of reductionism and new strategies of unification 


\begin{abstract}
Over the last decades, in biology there has been a proliferation of subdisciplines and theories, and this poses a question about its unity. Consequently, after reductionism was abandoned as a unifying strategy, philosophical questions have been raised about the possibility of alternative forms of relations among the subdisciplines, and whether they can effectively accomplish a unity of biology. This article starts with a brief consideration of some of the problems that the reductionist program confronts in biology, and then analyzes different kind of relations that are present among the subdisciplines of contemporary biology. In particular, we investigate four of the main proposals that can be found in the specialized philosophical literature; and then we propose two models of inter-disciplinary relations: isomorphism in evolutionary biology, and import/export of theoretical corpus, present in the relation between ecology and physiology. Finally, we offer a few ideas on unity as a goal for science in general, and also about the kind of cohesion that we think to be possible for biology based on our analysis. Thus, we defend the idea of pluralism of inter-disciplinary relations and of unity as a local phenomenon.
\end{abstract}

KEYWORDS $\bullet$ Unity of science. Reductionism. Inter-disciplinary relations. Subdisciplinary relations. Philosophy of Biology. Ecology.

\title{
REFERENGIAS BIBLIOGRÁFIGAS
}

Ayala, F. Introducción. In: Ayala, F. \& Dobzhansky, T. (Ed.). Estudios sobre la filosofía de la biología. Barcelona: Ariel, 1983. p. 9-29.

Ayala, F. \& Dobzhansky, T. (Ed.). Estudios sobre la filosofía de la biología. Barcelona: Ariel, 1983.

Beснтец, W. Integrating sciences by creating new disciplines: the case of cell biology. Biology and Philosophy, 8, p. 277-99, 1986.

. Discovering cell mechanisms: the creation of modern cell biology. Cambridge: Cambridge University Press, 2006.

BRIGANDT, I. Beyond reduction and pluralism: toward an epistemology of explanatory integration in biology. Erkenntnis, 73, p. 295-311, 2010.

Explanation in biology: reduction, pluralism, and explanatory aims. Preprint, 2011.

Brigandt, I. \& Love, A. Reductionism in biology. In: Salta, E. (Ed.). The Stanford Encyclopedia of Philosophy. Stanford: Stanford University Press, 2008. Disponível em: <http://plato.stanford.edu/entries/ reduction-biology/>. Acceso em: 20 mar. 2014.

Brown, J. \& MAURER, B. Macroecology: the division of food and space among species on continents. Science, 243, p. $1145^{-} 5^{\circ}, 1989$.

Brown, J.; Gillooly, J.; West, G. \& Savage, V. Toward a metabolic theory of ecology. Ecology, 85, p. 1771-89, 2004 .

Brown, J. \& MaUrer, B. Evolution of species assemblages: effects of energetic constraints and species dynamics on the diversification of the North American avifauna. American Naturalist, 13०, p. 1-17, 1987 .

BurIan, R. Underappreciated pathways toward molecular genetics as illustrated by Jean Brachet's cytochemical embryology. In: SARKAR, S. (Ed.). The philosophy and history of molecular biology: new perspectives. Dordrecht: Kluwer, 1996. p. 67-85.

CAPONI, G. La distinción entre biología funcional y biología evolutiva como clave para la discusión del reduccionismo en ciencias de la vida. Cadernos de História e Filosofia da Ciência, Série 3, 14, 1, p. 11957,2004 . 
Proliferagión subdisgiplinar en biología, debagle del RedugGionismo...

Cohen, R. et al. (Ed.). Boston studies in the philosophy of science. Dordrecht: Reidel, 1976. v. 30. (PSA1974).

DARDEN, L. Relations among fields: mendelian, cytological and molecular mechanisms. Studies in History and Philosophy of Biological and Biomedical Sciences, 36, p. 349-71, 2005.

Darden, L. \& Maull, N. Interfield theories. Philosophy of Science, 44, p. 43-64, 1977.

Di Pasquo, F. \& Folguera, G. Tres dimensiones del reduccionismo en el contexto de la teoría metabólica ecológica. Principia, 13, p. 51-65, 2009 .

Dobzhansky, T.; Ayala, F.; Stebbins, G. \& Valentine, J. Evolución. Barcelona: Omega, 1980.

Dupré, J. The disunity of science. Mind, 92, 367, p. 321-4,6, 1983. The disorder of things: metaphysical foundations of the disunity of science. Cambridge: Harvard University Press, 1993.

Gaston, K. \& Blackiburn, T. Pattern and process in macroecology. London: Blackwell, 2000.

Ginzburg, L. \& Colyvan, M. Ecological orbits. How planets move and population grow. Oxford: Oxford University Press, 2004.

Kitcher, P. $195^{3}$ and all that: a tale of two sciences. The Philosophical Review, 43, p. 335-71, 1984 . Unification as a regulative ideal. Perspective on Science, 7 , p. 337-48, 1999.

Love, A. Explaining evolutionary innovation and novelty: a historical and philosophical study of biological concepts (PhD dissertation). Pittsburgh: University of Pittsburgh, 2005.

Explaining evolutionary innovations and novelties: criteria of explanatory adequacy and epistemological prerequisites. Philosophy of Science, 75, p. 874-86, 2008.

Rethinking the structure of evolutionary theory for an extended synthesis. In: Pigliucai, M. \& MüLler, G. (Ed.). Evolution: the extended synthesis. Cambridge: The MIT Press, 2010. p. 4,03-4,2.

Maghamer, P.; Darden, L. \& Craver, G. Thinking about mechanisms. Philosophy of Science, 67, p. 1-25, 2000.

MAYR, E. Toward a new philosophy of biology. Cambridge: Harvard University Press, 1988.

NAGEL, E. The structure of science. London: Routledge and Kegan Paul, 1961.

Pigliugci, M. \& Kaplan, J. Making sense of evolution: the conceptual foundations of evolutionary theory. Chicago: University of Chicago Press, 2006.

Pigliucci, M. \& Müller, G. (Ed.). Evolution: the extended synthesis. Cambridge: The MIT Press, 2010.

Primas, H. Emergence in exact natural sciences. Acta Polytechnica Scandinavica, 91, p. 83-98, 1998.

Rosenberg, A. The structure of biological science. Cambridge: Cambridge University Press, 1985. Instrumental biology or the disunity of science. Chicago: University of Chicago Press, 1994.

SARKAR, S. (Ed.). The philosophy and history of molecularbiology: new perspectives. Dordrecht: Kluwer, 1996.

Wimsatт, W. Reductive explanation: a functional account. In: Conen, R. et al. (Ed.). Boston studies in the philosophy of science. Dordrecht: Reidel, 1976.v. 30, p. 671-710. (PSA-1974)

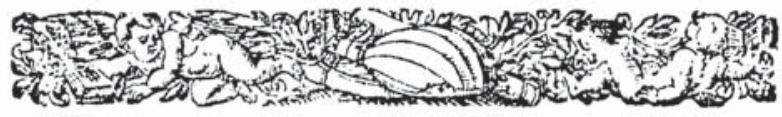

